\title{
DNA vaccines for viral diseases
}

J.J. Donnelly and J.B. UImer

\author{
Department of Virus and Cell Biology, Merck Research Laboratories, \\ West Point, PA, USA
}

\author{
Correspondence \\ J.J. Donnelly \\ Chiron Corporation \\ M/S 4.3156 \\ 4560 Horon St. \\ Emeryville, CA 94608 \\ USA \\ Fax: + 1-510-923-3229 \\ E-mail: john_donnelly@cc.chiron.com \\ Presented at the International \\ Symposium "The Third Revolution \\ on Vaccines: DNA Vaccines", \\ Belo Horizonte, MG, Brasil, \\ November 3-7, 1997. \\ ...................
}

Received October 5, 1998

Accepted October 30, 1998

\begin{abstract}
DNA plasmids encoding foreign proteins may be used as immunogens by direct intramuscular injection alone, or with various adjuvants and excipients, or by delivery of DNA-coated gold particles to the epidermis through biolistic immunization. Antibody, helper T lymphocyte, and cytotoxic $\mathrm{T}$ lymphocyte (CTL) responses have been induced in laboratory and domesticated animals by these methods. In a number of animal models, immune responses induced by DNA vaccination have been shown to be protective against challenge with various infectious agents. Immunization by injection of plasmids encoding foreign proteins has been used successfully as a research tool. This review summarizes the types of DNA vaccine vectors in common use, the immune responses and protective responses that have been obtained in animal models, the safety considerations pertinent to the evaluation of DNA vaccines in humans and the very limited information that is available from early clinical studies.
\end{abstract}

\section{Introduction}

Antibody, helper T lymphocyte, and cytotoxic $\mathrm{T}$ lymphocyte (CTL) responses have been induced in laboratory and domesticated animals by the direct inoculation of DNA plasmids encoding foreign proteins, either intramuscularly or by delivery of DNAcoated gold particles to the epidermis through biolistic immunization. In a number of animal models, immune responses induced by DNA vaccination have been shown to be protective against challenge with infectious agents. Immunization by injection of plasmids encoding foreign proteins has been used successfully as a research tool. This review summarizes the types of DNA vaccine vectors in common use, the immune responses and protective responses that have been obtained in animal models, the safety considerations pertinent to the evaluation of
DNA vaccines in humans and the very limited information that is available from early clinical studies.

\section{Construction of DNA vaccine plasmids}

Plasmids intended to be used as DNA vaccines contain the following essential elements: a strong eukaryotic promoter such as the immediate early promoter from cytomegalovirus; a gene of interest encoding an antigenic target expressed by a pathogen; a transcription terminator such as that from bovine growth hormone; an antibiotic resistance gene or other selectable marker to facilitate selection of transformed organisms carrying the plasmid, and an origin of replication, to allow for production of the plasmid in the desired host, that is not active in mammalian cells. Most commonly, plasmids 
of this type are produced in E. coli, using as a backbone an E. coli plasmid such as pBR322 or pUC18; in principle other hosts, e.g., yeasts, also could be used. The combination of the essential elements yields a eukaryotic expression vector that is capable of driving the production of the antigen of interest in cells of the vaccinated animal, but is unable to replicate in this species $(1,2)$.

It has generally been assumed that DNA vaccines that produce more antigen in situ will elicit higher levels of immune responses. Hence, DNA vaccine vectors have been developed to maximize the level of antigen expression. In general, strong viral promoters such as cytomegalovirus (CMV) and Rous sarcoma virus (RSV) have been employed and have proven effective in animal models. In systems where tissue-specific expression is desired, non-viral promoters may be considered. For example, the albumin promoter was used to target expression of antigen in hepatocytes by DNA vaccines, and the immunoglobulin promoter and enhancer elements were used to obtain preferential expression in B cells. All of the promoters listed above require that the expression vector be transported into the nucleus for transcription to take place. This requirement may be avoided by use of a bacteriophage $\mathrm{T} 7$ promoter system, where expression of the T7 RNA polymerase can drive expression of antigen controlled by the T7 promoter without need for host cell transcription machinery. This approach may also be useful for expressing proteins that require the involvement of other gene products (e.g., rev-dependent expression of HIV env).

\section{Immune responses induced by DNA vaccines}

\section{Humoral immunity}

Administration of plasmid DNA has proven to be an effective means of generating humoral immune responses against vari- ous foreign proteins in laboratory and domesticated animals and in some nonhuman primates. Antibody responses were first demonstrated in mice, induced by particle bombardment with gold beads coated with DNA encoding human growth hormone and human $\alpha-1$ antitrypsin (3). Antibodies against the viral influenza proteins nucleoprotein (NP) and hemagglutinin (HA) were demonstrated after im injection of DNA vaccines, as well as other antigens such as HIV envelope, bovine herpes virus glycoprotein, and hepatitis B surface antigen (2,4-8). Later, DNA vaccines were used to immunize animals against an idiotypic antibody of a Bcell lymphoma, carcinoembryonic antigen, human Ig V region, MHC class I molecules, rabies virus glycoprotein, herpes simplex glycoproteins B and D, HIV rev, papillomavirus $L 1$, hepatitis $C$ virus nucleocapsid, hepatitis B core protein, duck hepatitis B virus surface antigen, hepatitis $D$ and $E$ virus structural proteins, HTLV-1 envelope protein, St. Louis and Russian encephalitis virus prM/E, Japanese encephalitis virus NS1, dengue-2 envelope protein, CMV tegument pp65, bovine RSV G protein, foot-and-mouth disease virus, Schistosoma japonicum paramyosin, Plasmodium yoelii circumsporozoite protein, Leishmania major gp63, Bacillus thuringensis delta-endotoxin, fragment $\mathrm{C}$ of tetanus toxin, Brucella abortus ribosomal genes, Mycoplasma pulmonis, and Mycobacterium tuberculosis antigen 85 and hsp65 (9-13). Introduction of plasmid DNA into mucosal surfaces by feeding of plasmid encapsulated in microspheres, or by direct application of plasmid with mucosal adjuvants or cationic lipids, has been reported to yield secretory immune responses in mice. Because DNA vaccination results in the expression of antigen by host cells, proper antigenicity of proteins of eukaryotic origin in general, and of viral proteins in particular, may be achieved more readily than with other types of vaccines, such as inactivated viruses, subunits, or recombinant proteins. Therefore, DNA 
vaccination is an effective way of obtaining humoral immune responses in animals against viral, bacterial, parasitic, tumor, and eukaryotic proteins of immunological interest.

Early studies of antibody responses induced by DNA vaccines in animal models focused on viral envelope proteins, such as influenza HA, gD of herpes simplex virus, rabies virus glycoprotein, hepatitis B surface antigen ( $\mathrm{HbsAg}$ ), and HIV env, all of which are naturally expressed in membrane-anchored form. In the cases of influenza HA, HBsAg, and HIV env, in addition to the membrane-anchored form, a soluble form of the mature protein may also be generated naturally by enzymatic cleavage or by shedding of virus-like particles. Robust antibody responses, including conformationally specific virus-neutralizing antibodies, were readily demonstrated using these DNA vaccines. Later studies of DNA vaccines that encoded proteins that were not normally targeted for secretion, such as L1 of cottontail rabbit papillomavirus, showed that conformationally specific virus-neutralizing antibodies could be induced even though the protein product lacked secretion signal sequences. DNA vaccines that encode influenza NP also induce strong antibody responses, although the protein product lacks a conventional secretion signal sequence and contains a nuclear localization signal. DNA vaccines that encode secreted forms of soluble proteins, such as human growth hormone, immunoglobulin single-chain Fv's, or an N-terminal fragment of $\mathrm{HSV} g B$, have also been shown to be capable of eliciting antibody responses. In contrast, DNA vaccines that encode proteins that are degraded intracellularly, e.g., by ubiquitin-mediated proteolysis, produce strong cellular immunity but generally are ineffective or of much reduced effectiveness in inducing antibody responses (14). Thus, the ability of a DNA vaccine to produce antibody responses may depend more on its ability to produce mature protein in an appropriate conformation than on whether the protein is membrane-anchored or soluble, and whether it is targeted for secretion by conventional mechanisms. Thus, a reasonable approach to developing DNA vaccines against an unknown protein that is not normally secreted would be to express the protein both with and without an exogenous secretion signal sequence. For transmembrane proteins, expression of both fulllength membrane-anchored and truncated soluble forms would be appropriate.

\section{CD4+ $T$ cells}

CD4+ "helper" $\mathrm{T}$ cells are grouped into functional subsets characterized by the particular cytokines they produce. In mice, type 1-like helper (Th1) T cells predominantly produce the cytokines IL-2 and $\gamma$-interferon and support the development of cellular immune responses, including DTH (delayedtype hypersensitivity) and CTL, and antibody responses of the IgG2a immunoglobulin isotype. Type 2-like helper (Th2) T cells may produce IL-4, IL-5, IL-6, and IL-10, and promote B cell activation and immunoglobulin class switching. In mice, antibody responses driven by Th1-type T cells are predominantly of the IgG2a immunoglobulin isotype, while responses driven by Th2-type $\mathrm{T}$ cells are typified by a predominance of the IgG1 isotype. Intramuscular immunization of mice with DNA encoding a variety of antigens, such as influenza HA and NP, HIV env, gag, and rev, and M. tuberculosis antigen 85 , generates memory $\mathrm{T}$ lymphocyte responses (9). These have been identified both by antigen-specific $\mathrm{T}$ cell proliferation and by cytokine secretion during culture of splenocytes from vaccinated animals. Supernatants from antigen-stimulated cultures contained high levels of IL- 2 and $\gamma$-interferon with little or no IL-4 or IL-5, indicating that intramuscular DNA vaccination elicited Th1-like cytokine responses. In the studies of NP and env, selective removal of lymphocyte populations indicated that CD4+ T lym- 
phocytes were the primary responding cells in these assays. In contrast, vaccination by gene gun, which delivers DNA primarily to the epidermis, can lead to mixed-phenotype or Th2-like responses. It may be that the adjuvant effects of plasmid DNA tend to preferentially drive Th1 type responses in a dose-dependent manner; thus, intramuscular immunization, since it uses higher doses of DNA, may tend to preferentially activate Th1 cells.

\section{CD8+ T cells}

CD8+ cytotoxic $\mathrm{T}$ lymphocytes recognize peptides 8-10 amino acids in length that are displayed on the cell surface bound to MHC class I molecules. In general the peptides are generated in the cytosol by the action of the proteosome, the transporter of antigen peptide (TAP) gene products, or signal peptidase, and then transported to the endoplasmic reticulum where they bind to nascent MHC class I molecules. CD8+ CTL are present in lymph nodes and spleens of mice that have been injected intramuscularly with plasmid DNA encoding viral antigens, and can be demonstrated upon restimulation in vitro with antigen, or with mitogen and IL2 , or, in the case of LCMV, restimulation in vivo by viral infection. Effector CTL that recognized epitopes presented by class I molecules have been demonstrated in mice immunized with DNA encoding the NP from influenza A virus, HBsAg and core antigen, and HIV env $(2,9,11)$. CTL that were capable of recognizing and killing virus-infected targets have been induced in mice by DNA vaccines against influenza virus, against HIV (demonstrated using targets infected with vaccinia-HIVgp160 recombinants), against vaccinia virus or adenovirus-rabies virus glycoprotein recombinants, against LCMV, against HSV, and against measles virus. In studies of influenza NP in BALB/c mice, a single intramuscular injection of as little as $1 \mu \mathrm{g}$ of NP DNA induced CTL that recognized the 147-155 epitope peptide from influenza NP. Higher doses of NP DNA yielded CTL precursors at progressively increasing frequencies. Anti-NP CTL induced by im injection of influenza NP DNA were found to persist for more than two years. Subsequent im immunization and boosting with DNA vaccines increased cell-mediated immune responses to influenza NP and also to HIV env. Multiple im immunizations have been reported to drive immune responses toward a Th1 phenotype. In rhesus monkeys injected intramuscularly with DNA vaccines encoding either HIV env or gag genes, MHC class I-restricted, antigen-specific CTL developed following one or two vaccinations. Anti-env CTL were detected at least 11 months (the longest time point tested) following a final injection demonstrating that these responses can be long-lived (11).

Induction of CTL responses after gene gun immunization was first demonstrated using the gene for the MHC antigen $\mathrm{H}-2 \mathrm{~K}^{\mathrm{b}}$ administered directly into surgically exposed spleen and muscle. Mice immunized by these routes developed allospecific CTL. Studies of epidermal gene gun immunization in mice showed that 3 epidermal gene gun immunizations of $4 \mu \mathrm{g}$ each of a construct encoding HIV gp120 and $1 \mu \mathrm{g}$ each of a construct encoding HIV rev induced CTL responses that were detected after 2 immunizations but appeared to be suppressed by a third immunization, while antibody responses appeared after dose 3. This result may have been associated with a switch in helper $T$ cell phenotypes from Th1 to Th2, as the loss of CTL responsiveness was blocked by administration of antibody to IL-4. In rhesus monkeys, gene gun immunization using plasmids encoding SIV env and gag genes induced env-specific CTL in 3/3 monkeys, although gag-specific CTL were not detected. Intramuscular and intravenous injections of the same plasmids combined with gene gun immunization yielded env-specific CTL in $3 / 3$ animals and gagspecific CTL in 2/3 animals(15). 
Recent interest has focused on the potential mechanisms by which DNA vaccines induce CTL responses. The intramuscular injection of plasmid DNA transfects predominantly striated muscle cells, while epidermal immunization by gene gun may transfect epidermal keratinocytes and Langerhans cells, and also mononuclear cells present in epidermal capillary beds. Transplantation of C2C12 myoblasts stably transfected with the gene for influenza NP yielded NP-specific CTL responses, indicating that direct transfection of antigen-presenting cells (APC) is not required for the induction of CTL by DNA vaccines (16). However, studies in several laboratories have shown that bone marrow-derived APC are required for the induction of CTL by DNA vaccines administered intramuscularly, or epidermally by gene gun (17). Studies in our laboratory have shown that APC are required even when the NP protein is delivered by transplantation of stably transfected myoblast lines (16). Thus, the induction of CTL responses by DNA vaccines may occur, at least in part, by transfer of antigen from non-APC to APC (termed "cross-priming"). DNA vaccines consisting of plasmids encoding proteins targeted for intracellular degradation, or minigenes encoding minimal epitopes, are still capable of inducing CTL, and thus in the case of DNA vaccines cross-priming may occur by transfer of processed peptide as well as of the mature protein. Bone marrow-derived APC, such as dendritic cells, are potent inducers of CTL, and thus direct transfection of APC may also play an important role, e.g. in gene gun immunization.

\section{Protective immune responses in animal models}

Influenza was the first disease for which protective immunity induced by DNA vaccines was demonstrated in animal models. Cross-strain protection, in which mice immunized against one influenza subtype were rendered resistant to challenge by a different subtype, was induced by immunization with DNA encoding NP (2). In this system protection was obtained even though the immunizing antigen was taken from an influenza strain that arose 34 years before the challenge strain, and was of a different subtype. This illustrates the potential of CTL responses to provide broad cross-protection when highly conserved epitopes are targeted. Protective responses against homologous influenza strains were demonstrated in mice, chickens (5), and ferrets using DNA encoding HA. In ferrets, immunization with a combination of DNA plasmids encoding HA and two internal conserved proteins, NP and matrix, provided protection from an antigenically distinct isolate of influenza, as measured by a decrease in nasal viral shedding that was equivalent to protection provided by DNA encoding the homologous HA (4). Protective immune responses have been demonstrated in a number of other preclinical animal models including human immunodeficiency virus (LCMV) (18), bovine herpes virus, a murine and a mucosal guinea pig model of human herpes simplex virus, rabies virus, lymphocytic choriomeningitis virus, cottontail rabbit papilloma virus, hepatitis B virus, bovine respiratory syncytial virus, Japanese encephalitis virus, Russian spring/summer encephalitis virus, Central European encephalitis virus, and footand-mouth disease virus (9). While in most of the models immunity was dependent upon the generation of protective antibody responses, for LCMV and for the influenza studies with NP DNA the protection seen was based upon cellular responses.

\section{Safety considerations for use of DNA vaccines in humans}

Regulatory agencies charged with reviewing applications for the study of experimental DNA vaccines in humans have published "Points to Consider" documents that empha- 
size the following areas: potential integration of the plasmid DNA into the host cell genome; potential induction of autoimmunity or of immune tolerance, and potential induction of pathogenic anti-DNA antibodies.

\section{Potential for integration}

The integration of injected plasmid DNA into the genome of a living animal, if it occurred, might have no biological effect. Alternatively, such integration might potentially be mutagenic, if the integration event disrupted a cellular gene. In an extreme situation, integration might be carcinogenic, if the integration event were to inactivate a cell cycle regulatory gene or activate an oncogene. Integration by homologous recombination works most efficiently when both DNA copies are being replicated, and significant homology exists over a range of 600 base pairs. In the case of intramuscular DNA vaccination, the probability of integration appears to be very low. The plasmids commonly used for DNA vaccination do not contain an origin of replication that is functional in eukaryotic cells, the cells thought to be transfected (myocytes) are for the most part nondividing, and the plasmids used contain only very limited sequence homology with mammalian DNA. Studies of integration with sensitive PCR methods ( 1 copy/150,000 nuclei, which has been calculated to be 3 orders of magnitude less than the spontaneous mutation rate) in mice injected intramuscularly with plasmid DNA encoding influenza NP have not detected integration of the injected plasmid thus far (19).

\section{Potential for induction of immunologic tolerance and autoimmunity}

Since the amount of antigen produced after DNA immunization may be small, and since administration of small quantities of haptens or protein antigens can induce unre- sponsiveness, the formal possibility exists that antigen-specific unresponsiveness rather than protective immunity might be induced by DNA vaccines. However, many studies have demonstrated the ability of DNA vaccination to induce functional immune responses. Even in neonatal mice, injection of DNA vaccines may induce immunologic priming rather than unresponsiveness, depending upon the experimental system used (20). Autoimmune responses might be induced if the immune response to the encoded antigen results in immune-mediated destruction of the host cells expressing the antigen genes (21). However, the destruction of tissue cells occurs in normal processes of tissue remodeling as well as in the course of viral and bacterial infections. So far there is little evidence that DNA vaccines pose a greater risk in this regard than conventional viral or bacterial vaccines.

\section{Potential for induction of anti-DNA antibodies}

A third safety consideration concerns the potential for induction of pathogenic immune responses directed initially against the plasmid DNA itself. Although pathogenic anti-DNA antibodies are generally believed to be a hallmark of systemic lupus erythematosus (SLE), many other factors such as genetic susceptibility and underlying immune dysfunction play a role in these disease processes. It is not clear whether exposure to bacterial DNA can induce or exacerbate SLE in humans. Surprisingly, inoculation of spontaneously autoimmune mice with bacterial DNA can in some instances reduce the severity of their disease $(22,23)$. Although the likelihood of induction of pathogenic antiDNA antibodies by vaccination with plasmid DNA needs to be considered carefully, studies in laboratory animals have shown that purified dsDNA does not readily induce anti-DNA antibodies. To induce anti-dsDNA antibodies in normal mice it is necessary to 
inoculate them with DNA that has been denatured, complexed with methylated bovine serum albumin (mBSA) and coadministered with complete Freund's adjuvant (CFA). Injection of mice with dsDNA, regardless of origin, is not effective in inducing anti-DNA antibodies as measured by ELISA, immunoblot or radioimmunoassay (9). It is not yet known whether DNA vaccines will induce anti-DNA antibodies in humans.

\section{Phase I human clinical trials}

Clinical studies of DNA vaccines are still in very early stages. Phase I studies are ongoing in B cell lymphoma, HIV, influenza, malaria, herpes simplex, hepatitis $\mathrm{B}$, and melanoma. Little has been published to date but generally the vaccines appear to be well tolerated. Cell-mediated immune responses, including CTL, have been described in clinical studies of HIV (24) and malaria DNA vaccines (25). Antibody responses have been less widely reported. Some clinical responses (i.e., shrinkage of tumors) have been reported in studies of intratumoral injection of a plasmid encoding HLA-B7 formulated in cationic lipids. Further information on the dose range required to induce immune responses, the usefulness of various adjuvants and excipients, and the magnitude and duration of the resulting immune responses will be needed to assess the potential utility of DNA vaccines in humans.

\section{References}

1. Wolff J A, Malone RW, Williams $P$, Chong W, Acsadi G, J ani A \& Felgner PL (1990). Direct gene transfer into mouse muscle in vivo. Science, 247: 1465-1468.

2. Ulmer J B, Donnelly JJ, Parker SE, Rhodes GH, Felgner PL, Dwarki VJ, Gromkowski SH, Deck RR, Dewitt CM, Friedman A, Hawe LA, Leander KR, Martinez D, Perry HC, Shiver J W, Montgomery DL \& Liu MA (1993). Heterologous protection against influenza by injection of DNA encoding a viral protein. Science, 259: 1745-1749.

3. Tang DC, Devit M \& J ohnston SA (1992). Genetic immunization is a simple method for eliciting an immune response. Nature, 356: 152-154.

4. Donnelly JJ, Friedman A, Martinez D, Montgomery DL, Shiver J W, Motzel SL, Ulmer JB \& Liu MA (1995). Preclinical efficacy of a prototype DNA vaccine enhanced protection against antigenic drift in influenza-virus. Nature Medicine, 1: 583-587.

5. Fynan EF, Webster RG, Fuller DH, Haynes J R, Santoro JC \& Robinson HL (1993). DNA vaccines - protective immunizations by parenteral, mucosal, and gene-gun inoculations. Proceedings of the National Academy of Sciences, USA, 90: 11478-11482.

6. Cox G, Zamb TJ \& Babiuk LA (1993).
Bovine herpesvirus-1 - immune-responses in mice and cattle injected with plasmid DNA. J ournal of Virology, 67: 5664-5667.

7. Shiver J W, Ulmer J B, Donnelly JJ \& Liu MA (1996). Humoral and cellular immunities elicited by DNA vaccines: application to the human immunodeficiency virus and influenza. Advanced Drug Delivery Reviews, 21: 19-31.

8. Michel $M L$, Davis $H L$, Schleef $M$, Mancini M, Tiollais $P \&$ Whalen RG (1995). DNAmediated immunization to the hepatitis-B surface-antigen in mice - aspects of the humoral response mimic hepatitis-B viralinfection in humans. Proceedings of the National Academy of Sciences, USA, 92: 5307-5311.

9. Donnelly J J, Ulmer J B, Shiver J W \& Liu MA (1997). DNA vaccines. Annual Review of Immunology, 15: 617-648.

10. Barry MA, Lai WC \& J ohnston SA (1995). Protection against mycoplasma infection using expression-library immunization. Nature, 377: 632-635.

11. Liu MA, Yasutomi Y, Davies ME, Perry HC, Letvin NL \& Shiver J W (1996). Vaccination of mice and nonhuman primates using HIV gene-containing DNA. Antibiotics and Chemotherapy, 48: 100-104.

12. Stevenson FK, Zhu D, King CA, Ashworth LJ , Kumar S \& Hawkins RE (1995).
Idiotypic DNA vaccines against B-cell lymphoma. Immunological Reviews, 145: 211-228.

13. Wild J, Gruner B, Metzger K, Kuhrober A, Pudollek HP, Hauser H, Schimbeck R \& Reimann J (1998). Polyvalent vaccination against hepatitis B surface and core antigen using a dicistronic expression plasmid. Vaccine, 16: 353-360.

14. Rodriguez F, Zhang J \& Whitton J L (1997). DNA immunization: Ubiquitination of a viral protein enhances cytotoxic T lymphocyte induction and antiviral protection but abrogates antibody induction. J ournal of Virology, 71: 8497-8503.

15. Lu S, Arthos J, Montefiori DC, Yasutomi Y, Manson K, Mustafa F, J ohnson E, Santoro J C, Wissink J, Mullins J I, Haynes J R, Letvin NL, Wyand M \& Robinson HL (1996). Simian immunodeficiency virus DNA vaccine trial in macaques. J ournal of Virology, 70: 3978-3991.

16. Fu T-M, Ulmer J B, Caulfield MJ , Deck RR, Friedman A, Wang S, Liu X, Donnelly JJ \& Liu MA (1997). Priming of cytotoxic T lymphocytes by DNA vaccines: Requirement for professional antigen-presenting cells and evidence for antigen transfer from myocytes. Molecular Medicine, 3: 362371.

17. Doe B, Selby M, Barnett S, Baenziger J \& Walker CM (1996). Induction of cytotoxic 
T lymphocytes by intramuscular immunization with plasmid DNA is facilitated by bone marrow-derived cells. Proceedings of the National Academy of Sciences, USA, 93: 8578-8583.

18. Letvin NL, Montefiori DC, Yasutomi $Y$, Perry HC, Davies ME, Lekutis C, Alroy M, Freed DC, Lord Cl, Handt LK, Liu MA \& Shiver J W (1997). Potent, protective antiHIV immune responses generated by bimodal HIV envelope DNA plus protein vaccination. Proceedings of the National Academy of Sciences, USA, 94: 93789383.

19. Nichols WW, Ledwith BJ, Manam SV \& Troilo PJ (1995). Potential DNA vaccine integration into host cell genome. Annals of the New York Academy of Sciences, 772: 30-39.
20. Wang $Y$, Xiang $Z$, Pasquini $S \&$ Ertl $H C J$ (1998). Effect of passive immunization or maternally transferred immunity on the antibody response to a genetic vaccine to rabies virus. J ournal of Virology, 72: 17901796.

21. Davis HL, Millan CB \& Watkins SC (1997). Immune-mediated destruction of transfected muscle fibers after direct gene transfer with antigen-expressing plasmid DNA. Gene Therapy, 4: 181-188.

22. Gilkeson GS, Pippen AM \& Pisetsky DS (1995). Induction of cross-reactive antidsDNA antibodies in preautoimmune NZB/NZW mice by immunization with bacterial DNA. J oumal of Clinical Investigation, 95: 1398-1402.

23. Gilkeson GS, Ruiz P, Pippen AMM, Alexander AL, Lefkowith JB \& Pisetsky
DS (1996). Modulation of renal disease in autoimmune NZB/NZW mice by immunization with bacterial DNA. J ournal of Experimental Medicine, 183: 1389-1397.

24. Calarota S, Bratt G, Nordlund S, Hinkula J, Leandersson A-C, Sandstrom E \& Wahren B (1998). Cellular cytotoxic response induced by DNA vaccination in HIV-1-infected patients. Lancet, 351: 1320-1325.

25. Wang R, Doolan DL, Le TP, Hedstrom RC, Coonan KM, Charoenvit $Y$, J ones TR, Hobart $P$, Margalith M, NG J , Weiss WR, Sedegah $M$, de Taisne C, Norman J A \& Hoffman SL (1998). Induction of antigenspecific cytotoxic $T$ lymphocytes in humans by a malaria DNA vaccine. Science, 282: 476-479. 